\title{
Molecular imaging in nuclear cardiology: Pathways to individual precision medicine
}

\author{
A. Glasenapp, DVM, ${ }^{\mathrm{a}}$ A. Hess, PhD, ${ }^{\mathrm{a}}$ and J. T. Thackeray, $\mathrm{PhD}^{\mathrm{a}}$ \\ a Department of Nuclear Medicine, Hannover Medical School, Translational Cardiovascular \\ Molecular Imaging, Hannover, Germany
}

Received Jul 23, 2020; Revised Jul 29, 2020

doi: $10.1007 / \mathrm{s} 12350-020-02319-6$

Growth of molecular imaging bears potential to transform nuclear cardiology from a primarily diagnostic method to a precision medicine tool. Molecular targets amenable for imaging and therapeutic intervention are particularly promising to facilitate risk stratification, patient selection and exquisite guidance of novel therapies, and interrogation of systems-based interorgan communication. Non-invasive visualization of pathobiology provides valuable insights into the progression of disease and response to treatment. Specifically, inflammation, fibrosis, and neurohormonal signaling, central to the progression of cardiovascular disease and emerging therapeutic strategies, have been investigated by molecular imaging. As the number of radioligands grows, careful investigation of the binding properties and added-value of imaging should be prioritized to identify high-potential probes and facilitate translation to clinical applications. In this review, we discuss the current state of molecular imaging in cardiovascular medicine, and the challenges and opportunities ahead for cardiovascular molecular imaging to navigate the path from diagnosis to prognosis to personalized medicine. ( $\mathrm{J}$ Nucl Cardiol 2020;27:2195-201.)

Key Words: Positron emission tomography $•$ Cardiovascular disease • Inflammation • Fibrosis $\cdot$ Sympathetic nervous system

\begin{tabular}{ll} 
Abbreviations \\
FAP & Fibroblast activation protein \\
MI & Myocardial infarction \\
\hline
\end{tabular}

\section{INTRODUCTION}

As cardiovascular precision medicine embraces molecular-targeted therapies, the identification of at-risk and likely-to-respond patients takes on greater importance. Imaging to non-invasively quantify these molecular targets can provide incremental value in

Electronic supplementary material The online version of this article (https://doi.org/10.1007/s12350-020-02319-6) contains supplementary material, which is available to authorized users.

The authors of this article have provided a PowerPoint file, available for download at SpringerLink, which summarizes the contents of the paper and is free for re-use at meetings and presentations. Search for the article DOI on SpringerLink.com.

The authors have also provided an audio summary of the article, which is available to download as ESM, or to listen to via the JNC/ASNC Podcast. selecting appropriate patient populations for selective and expensive therapies. Accordingly, nuclear cardiology finds itself at a critical junction, where the pathway demarcated by image-guided oncology may direct the future of cardiovascular molecular imaging. Conventional nuclear cardiology assesses myocardial perfusion, viability, function, and scar-i.e., measurements of

Reprint requests: J. T. Thackeray, PhD, Department of Nuclear Medicine, Hannover Medical School, Translational Cardiovascular Molecular Imaging, Carl Neuberg Str 1, 30625 Hannover, Germany;

Thackeray.James@mh-hannover.de

J Nucl Cardiol 1071-3581/\$34.00

Copyright @ 2020 The Author(s) 


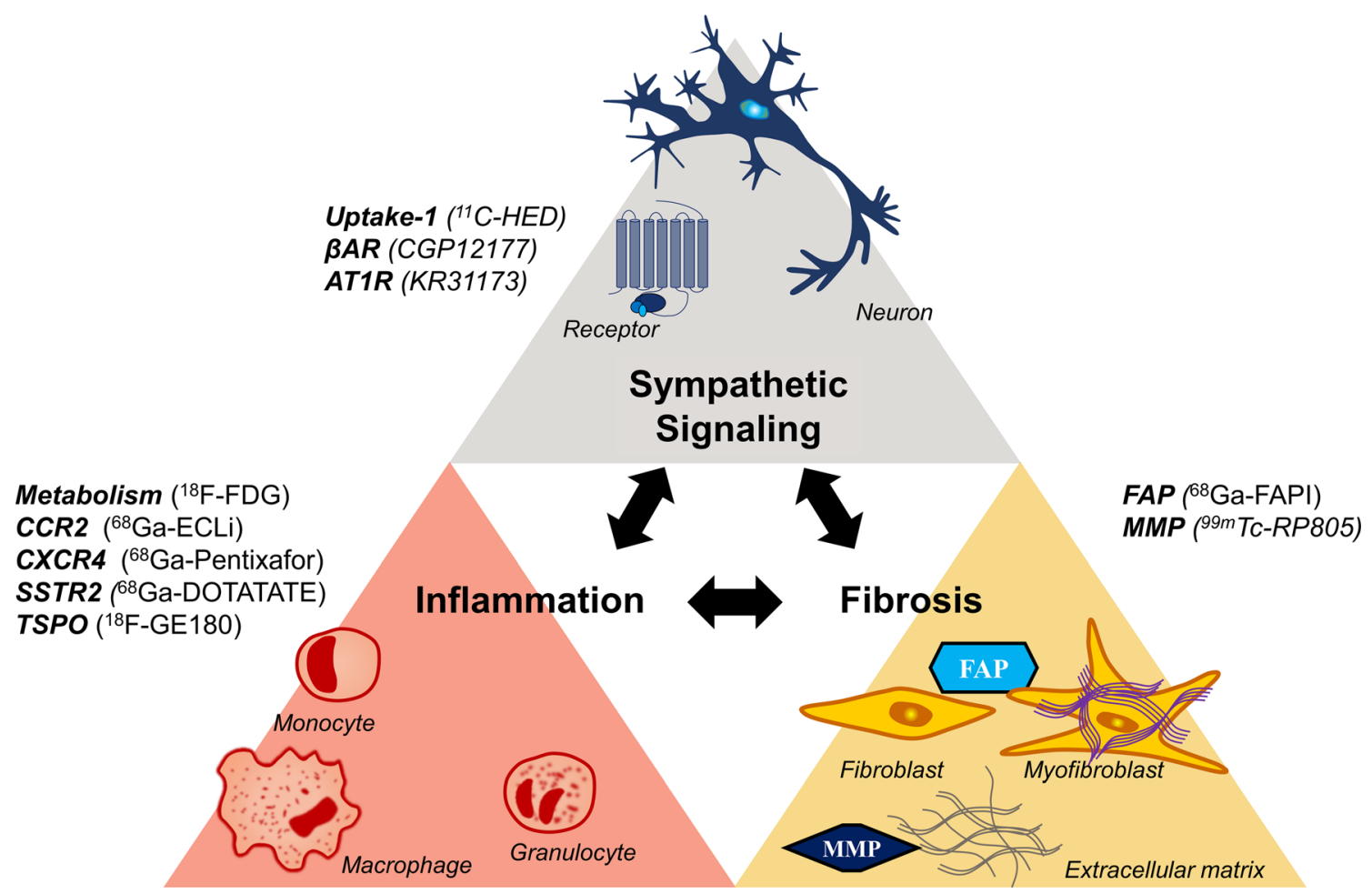

Figure 1. Overview of cardiovascular molecular imaging. Pathogenetic processes targeted by current radiopharmaceuticals include inflammatory leukocytes, fibroblasts, and proteases involved in matrix reorganization and sympathetic neuronal signaling. Each pathway is thought to influence the others by means of cytokines or signal transduction cascades.

disease severity after initial insult. ${ }^{1}$ However, these measures are generally observational and provide only limited opportunity for novel intervention, particularly at the molecular level. Accordingly, the development of new molecular-targeted imaging probes enables imaging at earlier stage of disease, building toward patient risk stratification, therapeutic guidance, and systems-based evaluations. To this end, the pathophysiological mechanisms of inflammation, fibrosis, and neurohormonal signaling have come to the forefront of molecular imaging in nuclear cardiology (Figure 1).

\section{Inflammation}

Inflammation critically contributes to development and progression of cardiovascular disease. After ischemic injury, cardiomyocyte death initiates release of pro-inflammatory factors, followed by leukocyte infiltration, remodeling, and repair. High circulating blood leukocytes are associated with higher mortality and adverse cardiac events among patients. ${ }^{2}$ Serumbased biomarkers, such as high sensitivity C-reactive protein, while widely used, are a crude indicator of local tissue inflammation, and accurate measurement of the injury microenvironment typically requires invasive biopsy. Molecular imaging enables a non-invasive 'virtual biopsy,' providing added-value in diagnosis and prognosis. Moreover, precise molecular therapies are emerging (e.g., antibodies and small peptides) which target specific components of the inflammatory pathway and bear potential to identify early pathological mechanisms for treatment to improve outcome. ${ }^{1}$ Early local inflammation after myocardial infarction (MI) in mice predicts functional outcome and provides guidance for precisely targeted and timed intervention. ${ }^{3}$

Contrary to the robust local inflammatory response after MI, non-ischemic cardiac diseases are characterized by diffuse myocardial inflammation, a greater challenge for imaging. The inflammatory response can be triggered by mechanical strain, neurohormonal activation, oxidative stress, fibrosis, and/or modest cardiomyocyte necrosis. ${ }^{4}$ Treatments typically minimize symptoms and improve quality of life, whereby blockbuster drugs delay or lessen remodeling but cannot avert disease progression. ${ }^{5}$ Early inflammation provides a therapeutic avenue which may complement 

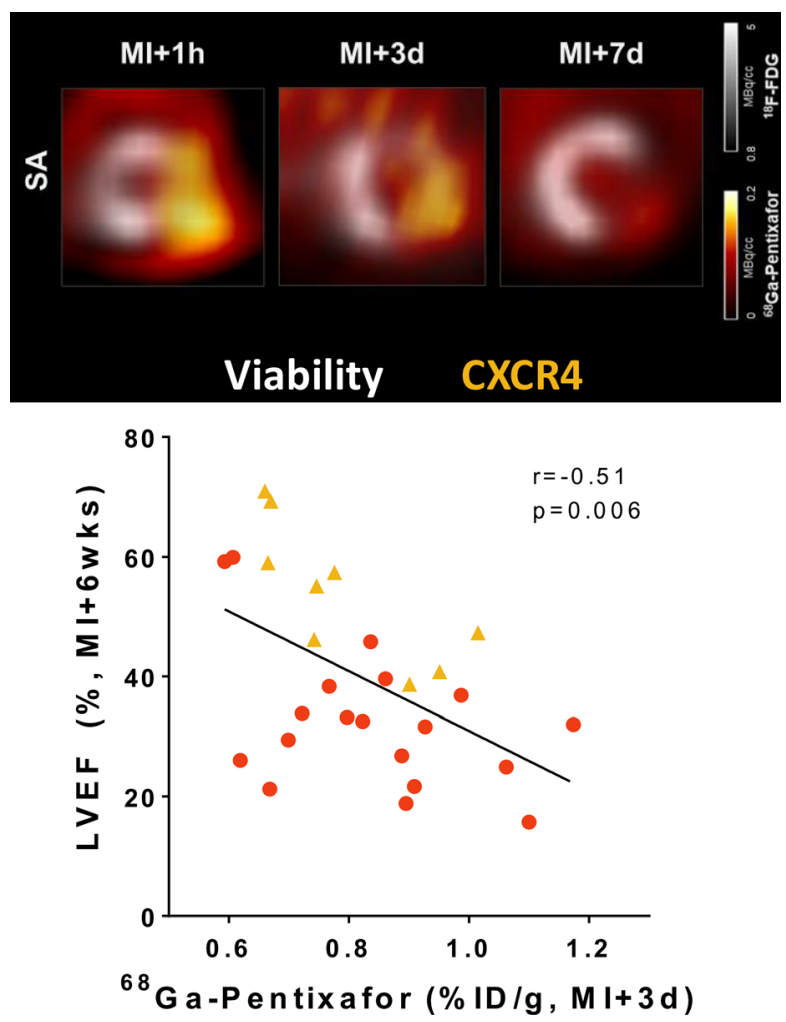

Figure 2. Molecular imaging of chemokine receptor CXCR4 after myocardial infarction. Transient upregulation of CXCR4 PET signal (colourscale) in the non-viable infarct zone (FDG, greyscale) at 1 hour and 3 days after coronary artery occlusion declines by 7 days in mice. The PET signal at 3 days predicts left ventricle ejection fraction (LVEF) 6 weeks later. Prepared using data from Hess et al. Eur Heart J 2020 ${ }^{3}$.

conventional therapy, such that precise characterization of the temporal and spatial inflammatory cell invasion can predict subsequent outcome (Figure 2). The presence of inflammation in atherosclerosis prior to coronary artery disease predicts future adverse cardiac events. ${ }^{6}$

As such, a number of molecular imaging agents have been explored for characterization of cardiac inflammation (Table 1). While most clinical experience relies on ${ }^{18} \mathrm{~F}$-fluorodeoxyglucose, novel molecular radioligands portend the opportunity to distinguish specific cellular components of the inflammatory response. ${ }^{7,8}$ These radioligands are most effective when coupled to specific therapies via the same molecular target, as for chemokine receptors. ${ }^{3,9}$

\section{Fibrosis}

Myocardial fibrosis is a common endpoint of cardiovascular disease, characterized by resident cardiac fibroblast transdifferentiation and activation, which

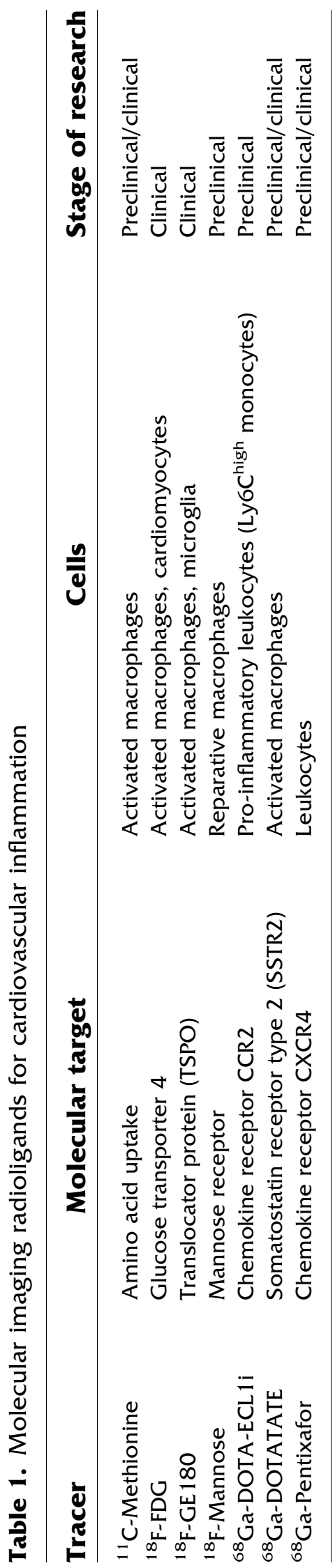


produce fibrillary collagen and reorganize extracellular matrix. Reparative or replacement fibrosis after ischemic injury culminates in scar formation and stabilization of the infarct. ${ }^{10}$ Reactive fibrosis is stimulated by local myocyte death, mechanical stimulus, or neurohormonal activation, leading to myofibroblast transdifferentiation and interstitial collagen deposition. ${ }^{11}$ The extended duration of the pathologic impetus, e.g., pressure or volume overload, cardiomyopathy, cardiotoxicity, infection, and metabolic stress, evokes prolonged myofibroblast activation, and progressive fibrogenesis over time. $^{12}$

Non-invasive characterization of fibrosis typically relies on estimation of ventricle stiffness and filling via echocardiography or characterization of tissue differences via cardiac magnetic resonance imaging. ${ }^{13,14}$ Prolonged T1 relaxation time on cardiac magnetic resonance imaging correlates to diffuse cardiac fibrosis in biopsy samples, ${ }^{15}$ suggesting the possibility to noninvasively characterize fibrotic burden in heart failure patients. But these measurements target the result of fibroblast activation, mature scar, or interstitial collagen late in disease progression. Accordingly, biomarkers of fibroblast activation early in pathogenesis are desirable. The fibroblast activation protein (FAP) is highly expressed by activated (myo)fibroblasts and is upregulated in response to ischemic and non-ischemic cardiomyopathy. ${ }^{16}$

To date, therapies to directly mitigate cardiac fibrosis are lacking, though novel strategies including gene transfer to reprogram cardiac fibroblasts ${ }^{17}$ or chimeric antigen $\mathrm{T}$ cells directed against $\mathrm{FAP}^{18}$ have shown promise in animal studies. Notably, conventional clinical management, including blockbuster drugs, slows the fibrotic mechanisms in hypertensive and heart failure patients, though the mechanism remains unclear. As such, visualization and quantification of early fibroblast activity provide insights into the pathology which may aid in drug development and optimization.

The expansion of imaging approaches for early indicators of fibrosis has stimulated interest in applying FAP-targeted imaging for cardiovascular disease. After permanent coronary artery ligation in rats, ${ }^{68} \mathrm{Ga}$-labeled FAP inhibitor accumulated in the infarct territory at $6 \mathrm{~d}$ after injury, receding to baseline subsequently. Signal specificity was confirmed by blocking and immunofluorescence staining. The density of FAP-positive fibroblasts was significantly higher in the infarct border zone compared to center or remote myocardium, ${ }^{19}$ suggesting the visualization of infarct expansion
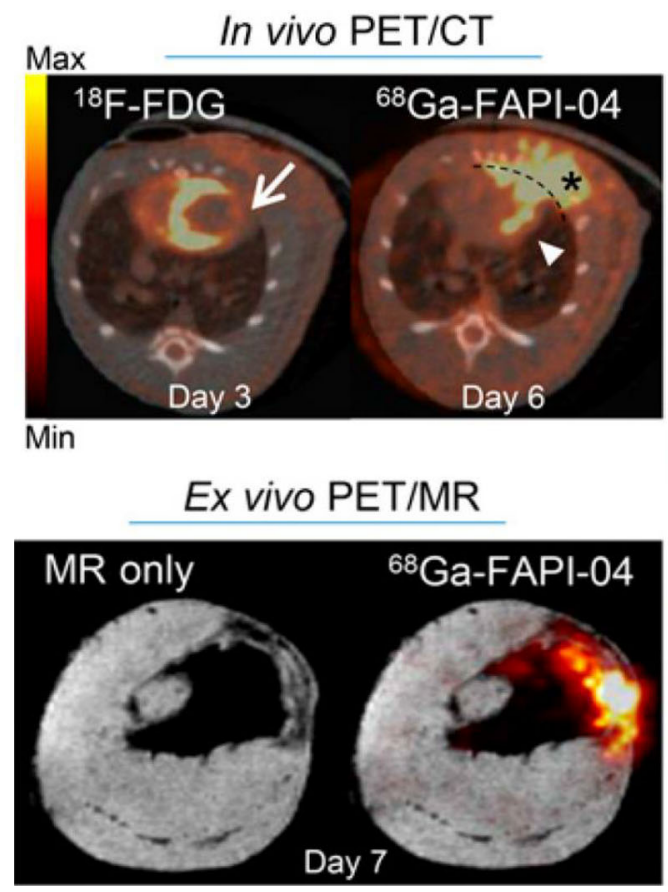

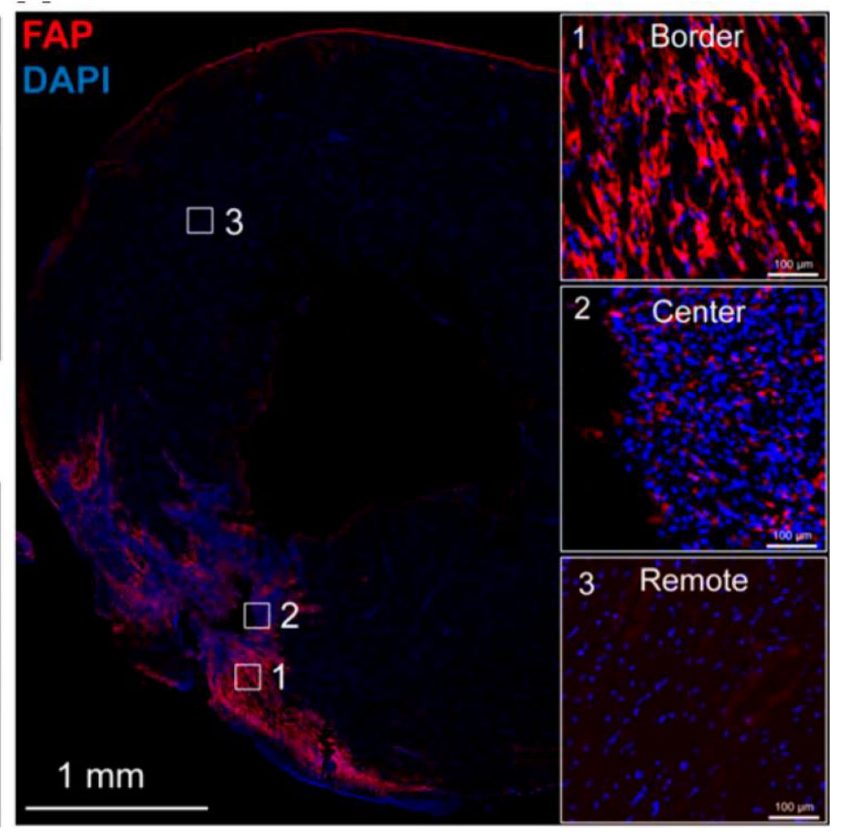

Figure 3. Visualization of fibroblast activation after myocardial infarction. Increased fibroblast activation protein (FAP) expression identified by ${ }^{68}$ Ga-FAPI-04 signal on PET-CT and ex vivo PET-MR in rats. Immunohistology confirmed high FAP expression in infarct border zone by myofibroblasts. Reproduced with permission from Varasteh et al. J Nucl Med. $201{ }^{19}$. 


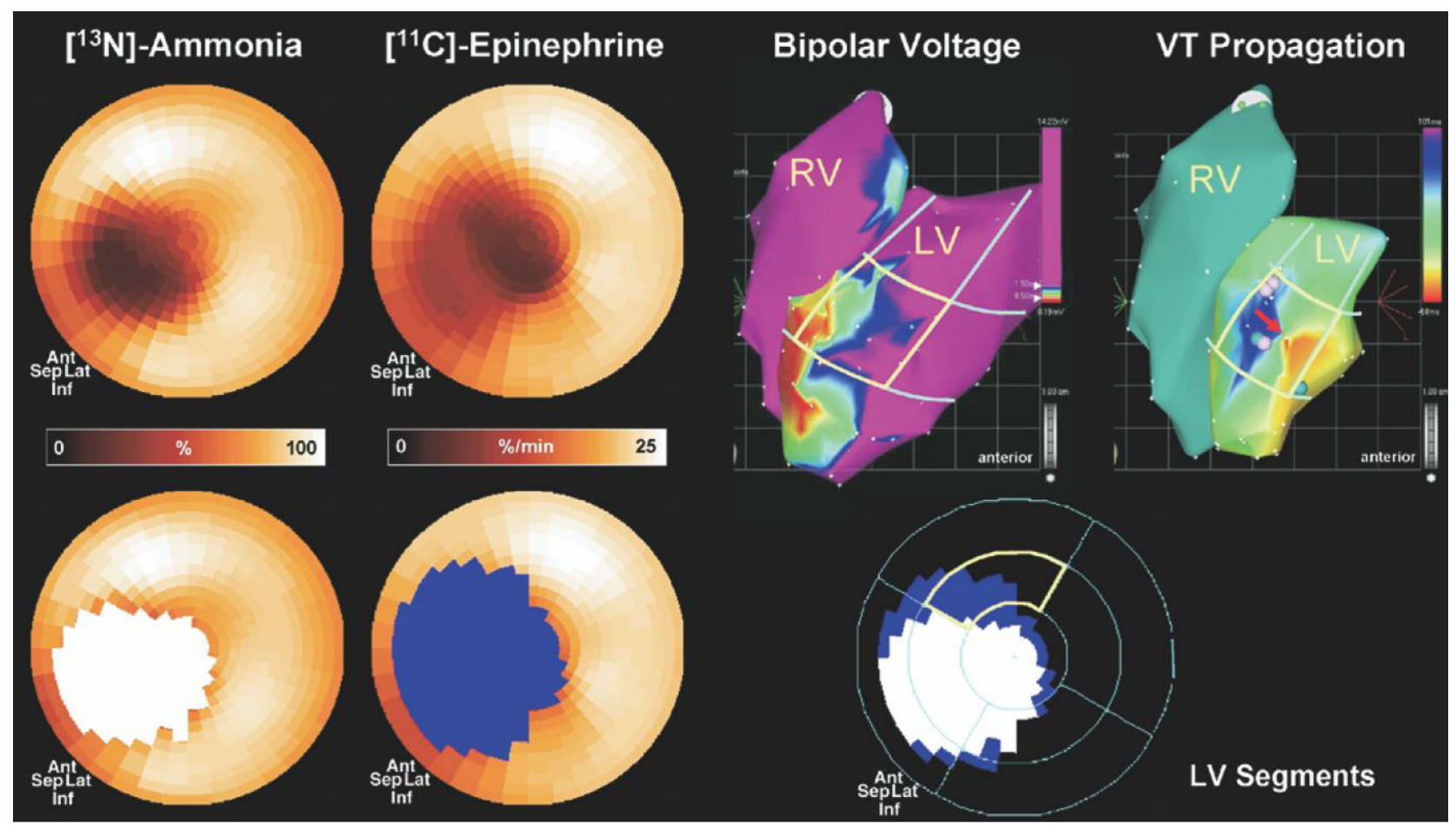

Figure 4. Imaging of cardiac sympathetic denervation identifies substrate of arrhythmia. Innervation defect defined by ${ }^{11} \mathrm{C}$-epinephrine exceeds the perfusion defect and colocalized to site of initiation of ventricular fibrillation on electrophysiology study after myocardial infarction in pigs. Reproduced with permission from Sasano et al. J Am Coll Cardiol. $2008^{21}$.

(Figure 3). Further research into prognosis and therapy response with FAP imaging and application in non-focal fibrotic disease is warranted.

\section{Neurohormonal Signaling}

The sympathetic nervous system is the primary extrinsic control of heart rate and contractility. Heightened sympathetic signaling compensates for the failing heart, leading to downregulation of adrenoceptors and excitation-contraction uncoupling. Beta-blocker therapy initially inhibits the over-stimulation of adrenoceptors, re-establishing homeostasis in autonomic regulation of contractile function. ${ }^{20}$ High sensitivity of sympathetic neurons to ischemia leads to selective dysinnervation of the heart after MI, which has been implicated as a substrate of ventricular arrhythmia and sudden cardiac arrest. $^{21,22}$

Imaging of the cardiac sympathetic nervous system has been pursued over the last three decades, but the impact on clinical care has been underwhelming. The principal limitation of innervation imaging lies with the radiotracers themselves, which are largely subject to variable permutations of neuronal reuptake, vesicular packaging, active synaptic release, passive diffusion to the synaptic cleft, and metabolic degradation. Despite evidence supporting the role of denervated myocardium in sudden cardiac arrest and heart failure progression (Figure 4), the limitations of quantification and tracer availability have prevented translation. Some of this hesitancy relates to the cost-effectiveness of imaging vs the fairly inexpensive cost of anti-adrenergic drugs. Newer compounds with favorable labeling and kinetics and targeting other signaling components such as angiotensin II type 1 receptors have been proposed, ${ }^{23,24}$ but have not yet seen widespread clinical application. Whether sympathetic neuronal imaging can be buoyed by these developments and connections to device therapy will ultimately determine its future.

\section{Challenges and Opportunities}

The expansion of the molecular imaging radiotracer arsenal provides a number of opportunities for research and patient management (Table 2). When these agents target pathogenetic mechanisms early in disease progression, they can facilitate risk stratification based on the expression pattern of inflammation, fibrosis, or 
Table 2. Challenges and opportunities for cardiovascular molecular imaging

Challenge

Tracer sensitivity

Prognostic value

Therapeutic response

Systems interaction

Pathway interface
Opportunity

Blocking studies for target specificity

Species differences in targets and affinity

Focal vs diffuse target expression

Test-retest reproducibility of signal

Quantitative tracer signal in disease models

Timecourse evaluation of disease-based signal (optimal timepoint)

Outcomes-based data to relate early signal to late function

Tracer sensitivity to therapeutic response

Timecourse evaluation of therapeutic response

Whole body analysis

Multi-tracer studies and timecourse evaluation sympathetic neuronal dysfunction at the site of injury. This approach allows regional organ interrogation at the site of injury and offers unique insight into pathobiology. Importantly, shared targets for imaging and therapeutic agents offer the potential to monitor early mechanisms of pathogenesis and direct clinical interventions toward patients at highest risk. Suitable patients and the optimal time point for treatment or intervention could be identified based on the temporal imaging signal.

To this end, targeted imaging and treatment of inflammatory and fibrotic mechanisms provide the opportunity to interrogate the intersection of these processes, which can further refine treatment strategies to benefit the individual patient. Moreover, the growing capacity to acquire images beyond the target organ, either through multiple bed positions or total-body PET, enables systems-based analysis, offering unique insights into the interaction of different organ systems. As such, cardiovascular molecular imaging can define the pathway to precision patient management, giving critical insights into disease processes, early prognosis, and response to therapy that can ultimately lead the right patient to the right therapy on the appropriate schedule.

\section{Disclosures}

All authors declare that they have no conflicts of interest. This work was supported by Deutsche Forschungsgemeinschaft KFO311 and TH2161/1-1.

\section{Funding}

Open Access funding provided by Projekt DEAL.

\section{Open Access}

This article is licensed under a Creative Commons Attribution 4.0 International License, which permits use, sharing, adaptation, distribution and reproduction in any medium or format, as long as you give appropriate credit to the original author(s) and the source, provide a link to the Creative Commons licence, and indicate if changes were made. The images or other third party material in this article are included in the article's Creative Commons licence, unless indicated otherwise in a credit line to the material. If material is not included in the article's Creative Commons licence and your intended use is not permitted by statutory regulation or exceeds the permitted use, you will need to obtain permission directly from the copyright holder. To view a copy of this licence, visit http://creativecommons.org/licenses/by/4.0/.

\section{References}

1. Hess A, Thackeray JT, Wollert KC, Bengel FM. Radionuclide Image-Guided Repair of the Heart. JACC Cardiovasc Imaging 2019.

2. Barron HV, Harr SD, Radford MJ, Wang Y, Krumholz HM. The association between white blood cell count and acute myocardial infarction mortality in patients $>$ or $=65$ years of age: findings from the cooperative cardiovascular project. J Am Coll Cardiol 2001;38:1654-61

3. Hess A, Derlin T, Koenig T, Diekmann J, Wittneben A, Wang Y et al. Molecular imaging-guided repair after acute myocardial infarction by targeting the chemokine receptor CXCR4. Eur Heart J 2020 (In Press).

4. Lindner D, Zietsch C, Tank J, Sossalla S, Fluschnik N, Hinrichs S, et al. Cardiac fibroblasts support cardiac inflammation in heart failure. Basic Res Cardiol 2014;109:428.

5. Frigerio M, Roubina E. Drugs for left ventricular remodeling in heart failure. Am J Cardiol 2005;96:10L-8L.

6. Figueroa AL, Takx RA, MacNabb MH, Abdelbaky A, Lavender ZR, Kaplan RS, et al. Relationship between measures of adiposity, 
arterial inflammation, and subsequent cardiovascular events. Circ Cardiovasc Imaging 2016;9:e004043.

7. Borchert T, Beitar L, Langer LBN, Polyak A, Wester HJ, Ross TL et al. Dissecting the target leukocyte subpopulations of clinically relevant inflammation radiopharmaceuticals. J Nucl Cardiol 2019.

8. Thackeray JT, Bengel FM. Molecular imaging of myocardial inflammation with positron emission tomography post-ischemia: A determinant of subsequent remodeling or recovery. JACC Cardiovasc Imaging 2018;11:1340-55.

9. Heo GS, Kopecky B, Sultan D, Ou M, Feng G, Bajpai G, et al. Molecular imaging visualizes recruitment of inflammatory monocytes and macrophages to the injured heart. Circ Res 2019;124:881-90.

10. Hara H, Takeda N, Komuro I. Pathophysiology and therapeutic potential of cardiac fibrosis. Inflamm Regen 2017;37:13.

11. Wynn TA, Ramalingam TR. Mechanisms of fibrosis: therapeutic translation for fibrotic disease. Nat Med 2012;18:1028-40.

12. Segura AM, Frazier OH, Buja LM. Fibrosis and heart failure. Heart Fail Rev 2014;19:173-85.

13. Moreo A, Ambrosio G, De Chiara B, Pu M, Tran T, Mauri F, et al. Influence of myocardial fibrosis on left ventricular diastolic function: noninvasive assessment by cardiac magnetic resonance and echo. Circ Cardiovasc Imaging 2009;2:437-43.

14. de Boer RA, De Keulenaer G, Bauersachs J, Brutsaert D, Cleland JG, Diez J, et al. Towards better definition, quantification and treatment of fibrosis in heart failure. A scientific roadmap by the Committee of Translational Research of the Heart Failure Association (HFA) of the European Society of Cardiology. Eur J Heart Fail 2019;21:272-85.

15. Kockova R, Kacer P, Pirk J, Maly J, Sukupova L, Sikula V, et al. Native T1 relaxation time and extracellular volume fraction as accurate markers of diffuse myocardial fibrosis in heart valve disease-comparison with targeted left ventricular myocardial biopsy. Circ J 2016;80:1202-9.

16. Tillmanns J, Hoffmann D, Habbaba Y, Schmitto JD, Sedding D, Fraccarollo D, et al. Fibroblast activation protein alpha expression identifies activated fibroblasts after myocardial infarction. J Mol Cell Cardiol 2015;87:194-203.

17. Song K, Nam YJ, Luo X, Qi X, Tan W, Huang GN, et al. Heart repair by reprogramming non-myocytes with cardiac transcription factors. Nature 2012;485:599-604.

18. Aghajanian H, Kimura T, Rurik JG, Hancock AS, Leibowitz MS, $\mathrm{Li} \mathrm{L}$, et al. Targeting cardiac fibrosis with engineered $\mathrm{T}$ cells. Nature 2019;573:430-3.

19. Varasteh Z, Mohanta S, Robu S, Braeuer M, Li Y, Omidvari N, et al. Molecular Imaging of fibroblast activity after myocardial infarction using a (68)Ga-labeled fibroblast activation protein inhibitor, FAPI-04. J Nucl Med 2019;60:1743-9.

20. Bristow MR, Ginsburg R, Minobe W, Cubicciotti RS, Sageman WS, Lurie K, et al. Decreased catecholamine sensitivity and betaadrenergic-receptor density in failing human hearts. N Engl J Med 1982;307:205-11

21. Sasano T, Abraham MR, Chang KC, Ashikaga H, Mills KJ, Holt DP, et al. Abnormal sympathetic innervation of viable myocardium and the substrate of ventricular tachycardia after myocardial infarction. J Am Coll Cardiol 2008;51:2266-75.

22. Fallavollita JA, Heavey BM, Luisi AJ Jr, Michalek SM, Baldwa S, Mashtare TL Jr, et al. Regional myocardial sympathetic denervation predicts the risk of sudden cardiac arrest in ischemic cardiomyopathy. J Am Coll Cardiol 2014;63:141-9.

23. Sinusas AJ, Lazewatsky J, Brunetti J, Heller G, Srivastava A, Liu $\mathrm{YH}$, et al. Biodistribution and radiation dosimetry of LMI1195: First-in-human study of a novel 18F-labeled tracer for imaging myocardial innervation. J Nucl Med 2014;55:1445-51.

24. Fukushima K, Bravo PE, Higuchi T, Schuleri KH, Lin X, Abraham MR, et al. Molecular hybrid positron emission tomography/computed tomography imaging of cardiac angiotensin II type 1 receptors. J Am Coll Cardiol 2012;60:2527-34.

Publisher's Note Springer Nature remains neutral with regard to jurisdictional claims in published maps and institutional affiliations. 